\title{
Boundary Vector Cells in the Subiculum of the Hippocampal Formation
}

\author{
Colin Lever, ${ }^{1}$ Stephen Burton, ${ }^{2}$ Ali Jeewajee, ${ }^{2,3}$ John 0'Keefe, ${ }^{2}$ and Neil Burgess ${ }^{3,4}$ \\ ${ }^{1}$ Behavioural Neuroscience Laboratory, Institute of Psychological Sciences, University of Leeds, Leeds LS2 9JT, United Kingdom, ${ }^{2}$ Department of Cell and \\ Developmental Biology, University College London (UCL), London WC1E 6BT, United Kingdom, ${ }^{3}$ UCL Institute of Cognitive Neuroscience, UCL, London \\ WC1N 3AR, United Kingdom, and ${ }^{4}$ UCL Institute of Neurology, UCL, London WC1N 3BG, United Kingdom
}

\begin{abstract}
"Boundary vector cells" were predicted to exist by computational models of the environmental inputs underlying the spatial firing patterns of hippocampal place cells (0'Keefe and Burgess, 1996; Burgess et al., 2000; Hartley et al., 2000). Here, we report the existence of cells fulfilling this description in recordings from the subiculum of freely moving rats. These cells may contribute environmental information to place cell firing, complementing path integrative information. Their relationship to other cell types, including medial entorhinal "border cells," is discussed.
\end{abstract}

\section{Introduction}

Stretching a familiar rectangular environment along one axis results in a stretching of place cell (PC) firing fields ("place fields") along the same axis (O'Keefe and Burgess, 1996). To explain this finding, we predicted "boundary vector cells" (BVCs) as inputs to the PCs (O'Keefe and Burgess, 1996, Burgess et al., 2000; Hartley et al., 2000). A BVC would fire whenever an environmental boundary intersected a receptive field located at a specific distance from the rat in a specific allocentric direction (Fig. 1A), with breadth of tuning to distance that increases with the preferred distance (Fig. 1B). The firing of a BVC depends solely on the rat's location relative to environmental boundaries and is independent of the rat's heading direction. BVCs with receptive fields peaked farther from the animal have broader firing fields than those peaked closer to it. Figure $1 C$ shows the BVC firing field (above) generated by a specific BVC receptive field (below).

The firing of a PC is a thresholded sum of the firing of the BVCs synapsing onto it. If a PC's input consists of a random selection of BVCs, then this model captures the statistics of the shape, number, and size of place fields as a function of the configuration of environmental boundaries (Hartley et al., 2000). Notably, the proportion of BVCs with a specific preferred distance has to decrease with preferred distance, so as to provide even coverage despite the increase in breadth of tuning (Hartley et al., 2000). As the environment becomes familiar, plasticity in the BVC-to-PC connections causes a "tidying" of PC firing, such that regions of lower firing rate are lost, whereas regions of higher firing rate strengthen (Barry and Burgess, 2007), consistent with

Received March 18, 2009; revised June 8, 2009; accepted June 29, 2009.

This work was supported by grants from the Medical Research Council, the Wellcome Trust, the Biotechnology and Biological Sciences Research Council, and European Union SPACEBRAIN. We thank Caswell Barry, Francesca Cacucci, and Tom Hartley for useful discussions and Tom Wills, Caswell Barry, and Julija Krupic for Matlab scripts.

Correspondence should be addressed to Colin Lever, John 0'Keefe, or Neil Burgess at the above addresses. E-mail: c.lever@leeds.ac.uk,n.burgess@ucl.ac.uk,orj.okeefe@ucl.ac.ac.uk.

D0I:10.1523/JNEUROSCI.1319-09.2009

Copyright $\odot 2009$ Society for Neuroscience $\quad$ 0270-6474/09/299771-07\$15.00/0 experimental data from CA1 (Lever et al., 2002b; Barry et al., 2006; Karlsson and Frank, 2008).

The power of the BVC model is seen in its ability to predict the effects of environmental manipulations on BVC and PC firing. For example, Figure $1 D$ shows the different spatial firing patterns expected of the BVC in Figure $1 C$ in four different environmental configurations, assuming that the sense of direction is held constant. Firing occurs at locations where the environmental boundary intersects the BVC's receptive field, producing crescent-shaped firing in the cylinder, firing parallel to one or more walls in a square box, and an additional firing field if a barrier perpendicular to the BVC's preferred direction is introduced into the environment. This second BVC field in response to insertion of a barrier should also be mirrored by the appearance of second fields in downstream PCs (Burgess et al., 2000), a prediction confirmed by Hartley et al. (2000) and Lever et al. (2002b). Plasticity in the BVC-PC connections (Barry and Burgess, 2007) satisfactorily models the disappearance of one of the two place fields once the configuration becomes familiar (Lever et al., 2002b; Rivard et al., 2004; Barry et al., 2006). Examining the effects of environmental shape on place fields was pioneered by Muller and Kubie (1987), whereas Sharp (1999) noted the importance of environmental boundaries for subicular firing. Here, we report cells recorded in the dorsal subiculum that fulfill the criteria for BVCs. Barry et al. (2006) presented a preliminary description of these cells.

\section{Materials and Methods}

Briefly, after surgical implants of microdrives loaded with platinumiridium tetrodes under anesthesia, we recorded from six rats with tetrodes located in the dorsal subiculum (rats 1-6; detailed methods in supplemental material, available at www.jneurosci.org). After electrode screening and foraging-task training in another room, each rat was brought to the testing laboratory and underwent a series of test trials, during which BVCs were recorded. Some BVCs were recorded in several environmental configurations. BVCs were also recorded after the environmental manipulations, in complete darkness or on the holding platform. We took care to anchor the head-direction 

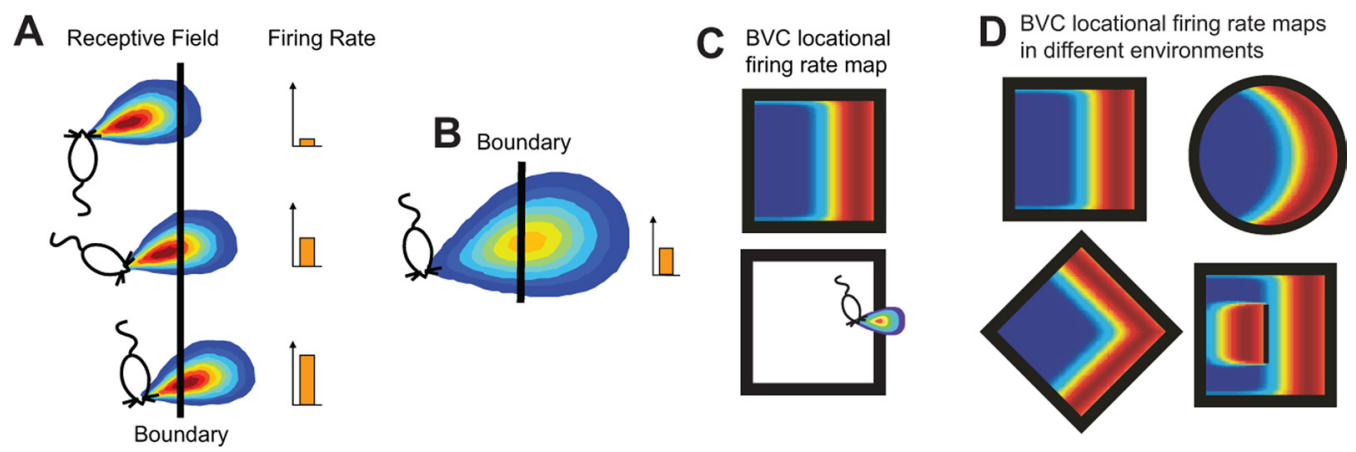

Figure 1. The BVC model. A BVC responds maximally when a boundary is perceived at a preferred distance and allocentric direction from the animal, regardless of the animal's heading direction. $\boldsymbol{A}$, The receptive field of a BVC tuned to respond to a barrier at a short distance east-northeast from the animal. $\boldsymbol{B}, \mathrm{BVCs}$ tuned to respond to barriers farther from the animal will have broader receptive fields. C, The firing field (firing rate as a function of the animal's location; top) for a BVC with a receptive field tuned to respond to a boundary at a short distance to the east (bottom). D, Predicted firing fields in different environments for the BVC shown in C. Insertion of a barrier causes a doubling of the field (bottom right panel). Figures are adapted from Hartley et al. (2000) and Barry and Burgess (2007).

system across all testing environments (see supplemental Methods, available at www.jneurosci.org as supplemental material).

\section{Results}

\section{Dataset of BVCs}

Multiple single units were recorded in 10 min trials of foraging in a "standard" recording environment (environment a) consisting of a square box with 50 -cm-high walls (of beige "morph box" material) (Lever et al., 2002a) set within a black-curtained arena containing a white cue card. Thirty-six putative BVCs had spatial firing fields consistent with a single receptive field (Fig. 2). Following Cacucci et al. (2004) and Solstad et al. (2008), we estimate an upper bound on the proportion of BVCs in the population by noting that, in trials in which 45 recordings of BVC-like firing fields were made ( 9 of the 36 BVCs being resampled on subsequent days), 141 non-BVC firing fields were also recorded. Thus, up to $24 \%$ ( 45 of 186) of cells in this region are BVCs.

\section{Firing fields of BVCs in the standard recording environment (square box)}

Figure 2 shows the firing fields of $36 \mathrm{BVCs}$ recorded in the standard square box. Purely to illustrate the consequences of variance in preferred distance tuning and preferred allocentric directional tuning upon the expression of spatial firing fields in this environment, we have divided the cells in Figure 2. We assume that tuning to distance and direction varies continuously within the BVC population, albeit with a bias toward shorter distances (Hartley et al., 2000). BVCs with receptive fields peaked close to the animal and oriented perpendicular to the walls of the box fire predominantly when the animal is close to the wall in the preferred direction (Fig. 2A,B). BVCs with directional preferences between the perpendiculars to adjacent walls fire along both walls (Fig. 2C,D). BVCs with receptive fields peaked farther from the animal's head have firing fields that are broader but may still lie directly against a wall (Fig. $2 B, D$ ), although if peaked far enough from the animal, the firing field will be offset from the wall (Fig. $2 E$ ). Large firing fields may be BVCs peaked far from the animal and responding to distant rather than proximal walls (Fig. $2 F$ ).

\section{Firing to different types of boundary and insensitivity to context}

We tested 24 of these BVCs in environments additional to the standard recording environment (environment a) (see Fig. 3 and supplemental Methods, available at www.jneurosci.org as supplemental material, for details). Seventeen cells were tested in a cylinder with 50-cm-high gray walls and with room cues visible (environment $\mathrm{b}$ ) and in an environment consisting of the raised, circular floor of environments $\mathrm{a}$ and $\mathrm{b}$ (bounded by a drop, rather than walls; environment c) (Fig. $3 A, B$ ). Seven cells were tested in circular and diamond configurations of the morph box used in environment a (Fig 3C). Of these 24 cells, 11 were also recorded on the raised holding platform, located $2 \mathrm{~m}$ outside of the usual recording arena (environment d) (Fig. $3 A, C)$, and 3 were also recorded in larger environments with and without an additional internal boundary (Fig. 3D,E).

In general, the firing fields of BVCs were a function of a distance and allocentric direction vector to the boundaries of the local environment. The long axes of firing fields reflected the curvature of the boundaries, tending to be straight in square and curved in circular environments (Fig. 3A, cells 5c, 2a, 5e, and 5a). Unlike the place fields of PCs that "remap," the presence of BVC firing fields was independent of the color, shape, and material of the walls bounding an enclosure or the distal cues behind it (compare Fig. $3 A-C$, environments a and b). Even the dramatic change in boundary from an enclosure wall to a drop over an edge had a limited effect on firing patterns (Fig. $3 A$, environment c). In contrast, CA1 PCs (five rats, including four that had simultaneous CA1/subiculum recordings) show strong remapping between environments a-c: briefly, spatial bin-by-bin correlations were high between the two environment a trials (mean $r=0.803$ ) but very low between environment $\mathrm{a}-\mathrm{b}$ trials (mean $r=0.053$ ) and lower still between environment a-c trials (mean $r=-0.049$ ) (C. Lever, unpublished observation). Thus, BVC firing is consistent across different environments, conforms to the predictions of the model, and differs from PCs recorded in the same situation.

The predicted doubling of the firing field in response to insertion of an appropriately oriented barrier (Fig. 1D) is demonstrated in three BVCs (Fig. 3D,E). Cell 1a also responded to the boundary created by the creation of an edge when two platforms (Fig. 3E, e Together) were pulled apart to create a $13 \mathrm{~cm}$ gap between them, extending the full width of the environment (Fig. $3 E$, e Apart). The rat voluntarily crossed this boundary four times and was manually carried across five times, with the same results. The cell treated this split environment as containing two southern boundaries and exhibited two firing fields akin to barrierelicited field doubling. 


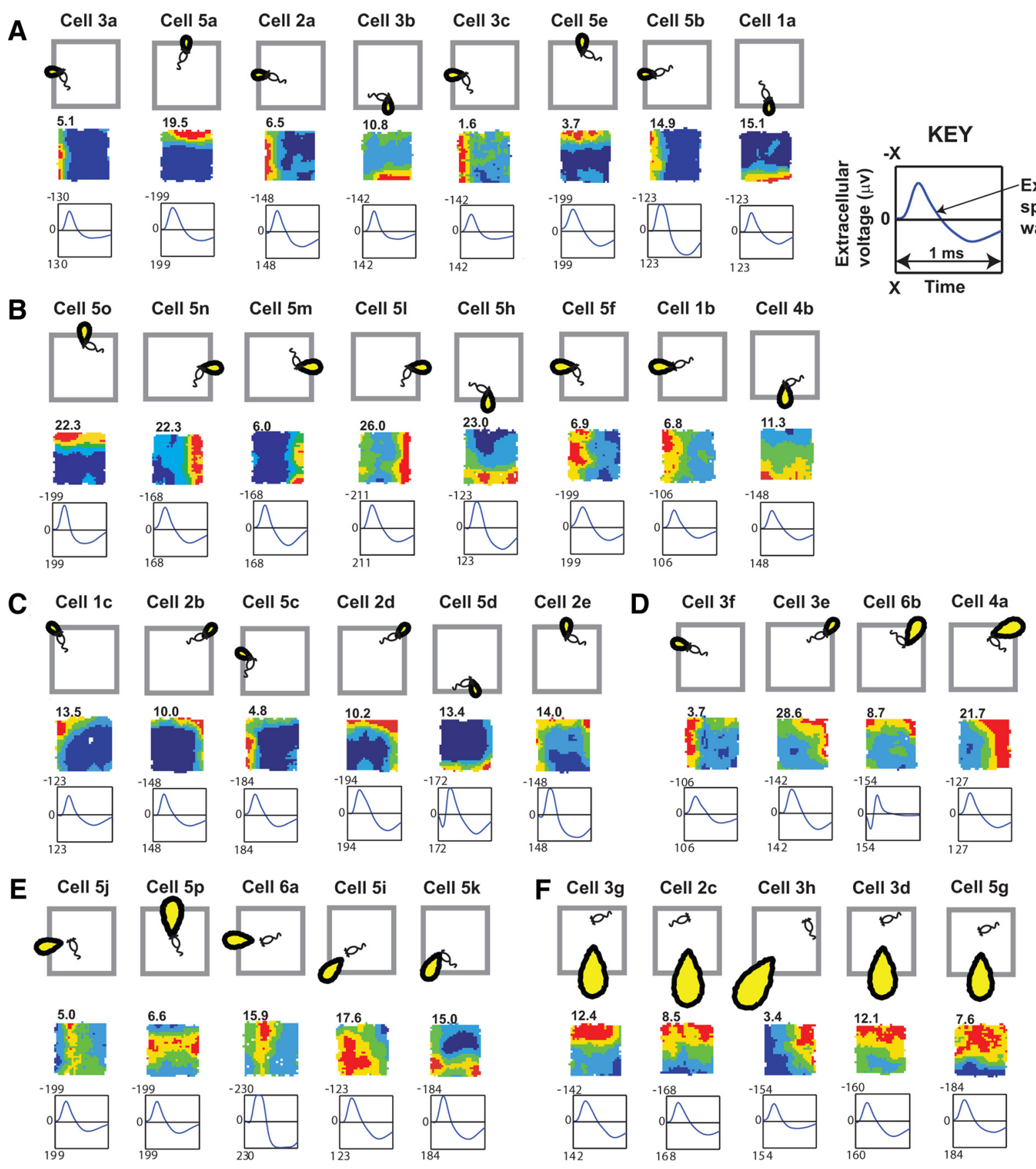

Figure 2. Putative BVCs recorded from dorsal subiculum. Firing fields (middle rows) from trials in environment a and corresponding BVC receptive fields (top rows). Cells are identified by animal number, followed by cell letter. The number shown top left of the firing rate map denotes peak rate in hertz after smoothing [firing rate map methods as in the study by Wills et al. (2005)]. The bottom rows show the averaged waveform taken from the tetrode channel with the largest peak-to-trough amplitude: $y$-axis is extracellular voltage (in microvolts, negative upward); $x$-axis is time (1 ms).

As well as wall-like boundaries, a sheer drop (environments c and e Apart) or a low ridge followed by a drop (environment d) also form boundaries that can anchor BVC firing (Fig. 3A). These wall-less environments actually provided two parallel sets of boundaries: (1) the platform edge; and (2) $\sim 5-10 \mathrm{~cm}$ farther out, the limit of the space reachable by leaning over the edge. (The outer limit of space reached by the animal's head is shown by the outer limit of the firing rate maps.) BVC responses to both types of boundary can be seen in Figure $3 A$, by comparing firing in wall-less environments $\mathrm{c}$ and $\mathrm{d}$ with firing in the walled environments a and b. Some BVCs appear to respond predominantly relative to the platform edge (Fig. $3 A$, cells $5 c, 5 e, 5 k$, and $4 b$ ) and others predominantly relative to the edge of reachable space (Fig. $3 A$, cells $2 a, 1 a, 5 a$, and $6 \mathrm{~b}$ ). The more diffuse firing fields in some cells in the wall-less environments (Fig. $3 A$, cells $2 \mathrm{a}, 1 \mathrm{a}, 1 \mathrm{~b}$, and $4 \mathrm{a}$ in environment c) could reflect firing driven by both types of boundary.

Recordings made in complete darkness in the standard environment (Fig. $3 C$ ) indicate that visual input is not necessary to drive BVCs. The 11 BVCs recorded on the holding platform (environment $\mathrm{d} ; 2 \mathrm{~m}$ from the main recording arena) all showed BVC-like responses (Fig. $3 A, C$ ), notwithstanding the more diffuse firing pattern, indicating that BVC firing is independent of testing location within the experimental room. 

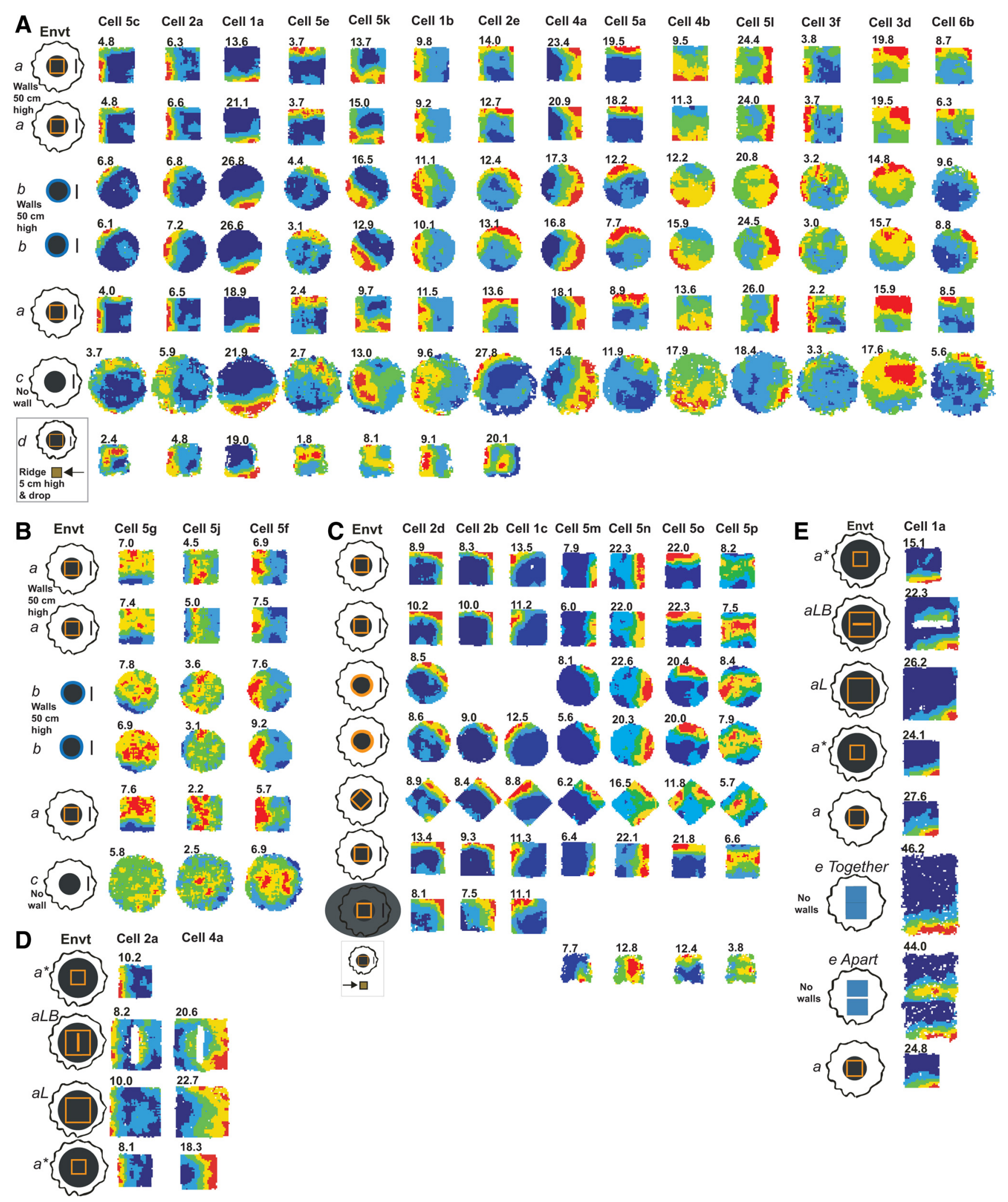

Figure 3. BVC firing fields in different environments. $A$, Firing fields of 14 subicular BVCs in different environments a- $d$ (see supplemental material, available at www.jneurosci.org, for a full description). Environment (Envt) a, $62 \times 62 \times 50$-cm-high beige square box made of morph material; Envt b, 79-cm-diameter, circular-walled, wooden light-gray enclosure; Envt c, the 90-cm-diameter floor of Envts a and b; Envt d, 39-cm-sided, square holding platform with 5-cm-high ridges located $2 \mathrm{~m}$ south of Envt a. $B$, Firing fields of three BVCs in environments a- $c$, which have more diffuse firing in Envt c than those shown in $A$. C, Firing fields of seven BVCs tested in square-, circular-, and diamond- shaped environments (all made of the same morph box material). Three of these were also recorded in complete darkness (row 7), and four were recorded on the holding platform (Envt d, row 8). D, E, Cells 2a and 4a (D) and cell 1a (E) under additional environmental manipulations. Envt a* is Envt a placed on a larger platform; Envts aL and aLB are larger square environments made of the same morph material (aLB also contains a barrier). In the e Apart condition, a $13 \mathrm{~cm}$ gap is interposed between the two elevated, rectangular "drop" platforms of the e Together condition. Cells $2 \mathrm{a}$ and $5 \mathrm{c}$ are adapted from Barry et al. (2006). 

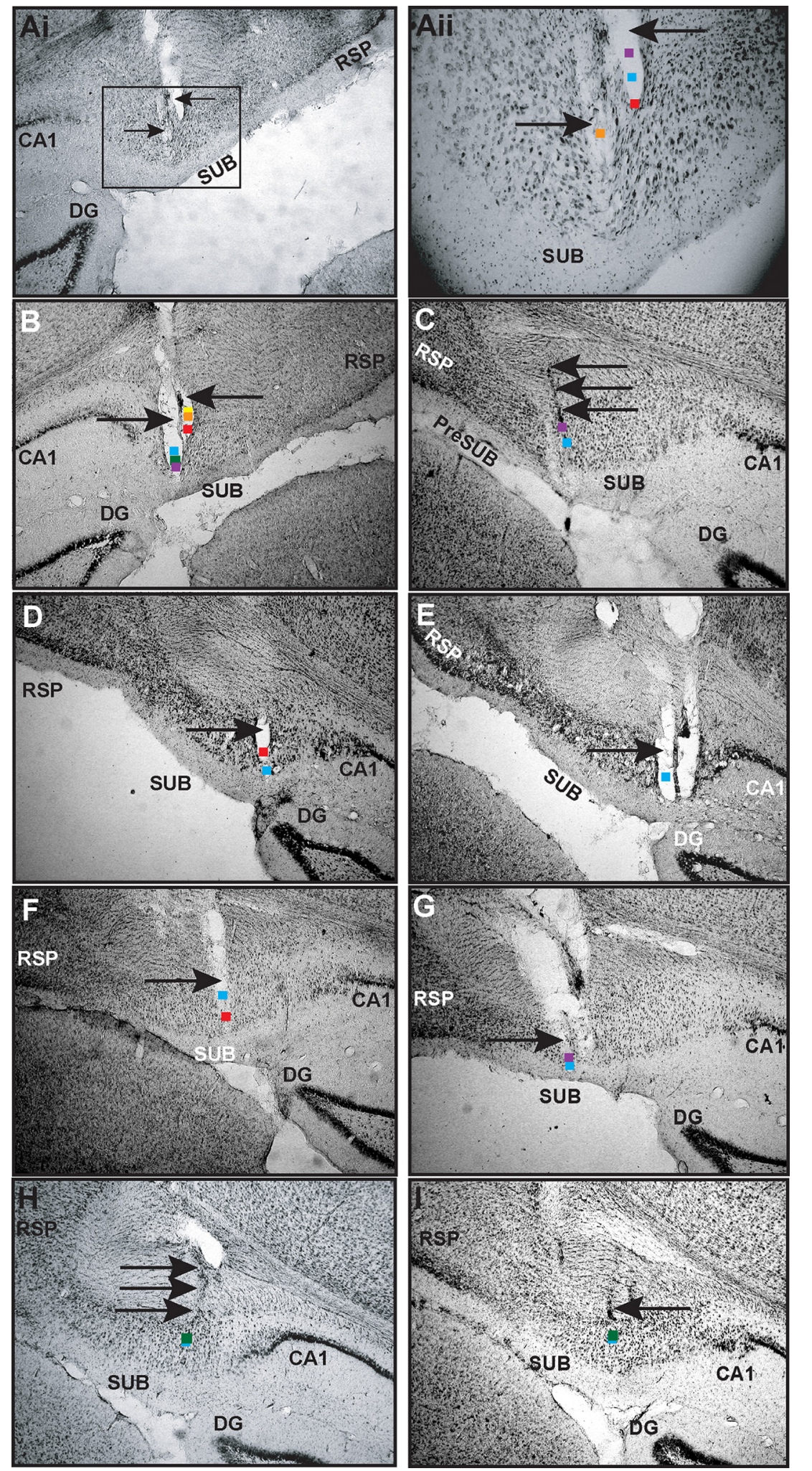

Figure 4. Recording locations of BVCs. Nissl-stained sections of the dorsal subiculum are shown. Colored squares indicate estimated locations of BVCs; arrows indicate tracks of recording tetrodes. Cells recorded at depths $50 \mu \mathrm{m}$ apart are shown at the same location. Ai, Tetrode tracks in subiculum of rat 5. Aii, Close-up of the rectangular area indicated in Ai. Orange square, Estimated location of cell 5j; purple square, cell 5 h; blue square, cell 5 ; red square, cell $5 \mathrm{~m}$-n. $B$, Purple square, cell 50-p; green square, cells $5 a, 5 e, 5 f$, and $5 k$; blue square, cell $5 \mathrm{~d}$; red square, cell 5 ; ; orange square, cell 51 and 5 b; yellow square, cell 5 i. C, Purple square, cell 6a; blue square, cell 6b. D, Red square, cell 1a; blue square, cell 1c. $E$, Blue square, cell 1b. $F$, Red square, cells 2a and 2e; blue square, cell 2c. G, Blue square, cell $2 \mathrm{~b}$; purple square, cell $2 \mathrm{~d}$. $\boldsymbol{H}$, Green square, cells $3 \mathrm{a}$, $3 \mathrm{e}$, and $3 \mathrm{~g}$; blue square, cell $3 \mathrm{~d}$. The estimated location of cell $3 \mathrm{~h}$ is similar to cells $3 \mathrm{3a}, 3 \mathrm{e}$, and $3 \mathrm{~g}$, but $90 \mu \mathrm{m}$ posterior (data not shown). I, Green square, cell $3 \mathrm{~b}$-c; blue square, cell 3f. SUB, Subiculum; RSP, retrosplenial cortex; DG, dentate gyrus.

\section{Boundary-related firing is} not directional

A key prediction of the BVC model is that BVCs code for distances to boundaries in allocentric directions independent of the directional orientation of the rat. To test this, we examined the directionality of the 24 BVCs tested in the cylindrical environments (environment $\mathrm{c}$ or morph circle) using firing rate maps and polar plots corrected for spurious dependencies created by inhomogenous sampling of orientation and location (see supplemental material, available at www.jneurosci.org). Estimated mutual information between firing rate and location was $0.25 \pm 0.05$ bits per spike (mean \pm SEM) and $0.05 \pm$ 0.01 bits per spike between firing rate and direction. Thus, BVC firing contains five times more locational than directional information. In addition, BVCs show very low "directional selectivity" of firing (maximum firing rate across $60 \mathrm{di}$ rectional bins divided by mean rate): $1.56 \pm 0.05$. This is less than the directional selectivity of presubicular headdirection cells (selectivity, $6.91 \pm 0.05$; $n=45$ ) (Cacucci et al., 2004) or even CA1 PCs (selectivity, $2.87 \pm 0.20 ; n=$ 46) (Cacucci et al., 2004) recorded and analyzed similarly (supplemental material, available at www.jneurosci.org).

\section{BVCs are recorded from dorsal} subiculum and have extracellular spike waveforms characteristic of principal cells

Our tetrodes targeted a restricted region of the dorsal subiculum $(5.8-6.3 \mathrm{~mm}$ behind bregma). Figure 4 indicates the estimated recording locations in the dorsal subiculum for 34 of the 36 BVCs shown in Figure 2. Briefly, BVCs are found in both proximalto-CA1 and distal-to-CA1 portions of the dorsal subiculum and in deep and superficial layers of the subiculum pyramidal cell layer, with no obvious preferential distribution; we found more BVCs in proximal-toCA1 subiculum (26 of 36 in proximal half), but this simply reflects electrode sampling bias (see supplemental material, available at www.jneurosci.org). The extracellularly recorded waveforms of BVCs from the channel showing the largest peak-totrough amplitude had mean peak-totrough latencies of $474 \pm 8 \mu$ s and mean peak-to-trough amplitudes of $213 \pm 12 \mu \mathrm{V}$ (Fig. 2A-E, bottom rows). These long peakto-trough latencies and high peak-totrough amplitudes are consistent with BVCs being principal cells (pyramidal cells in the subiculum). See Solstad et al. (2008) for similar measurements in entorhinal cortex. 


\section{Temporal firing characteristics of BVCs}

Temporal firing characteristics of the 36 BVCs were as follows (for details, see supplemental Methods, available at www.jneurosci. org as supplemental material): global firing rate, $5.04 \pm 0.59 \mathrm{~Hz}$ (range, $0.56-13.3 \mathrm{~Hz}$ ); theta modulation, $10.0 \pm 1.5$ (range, 1.338.8); modal interspike intervals (ISIs), $33.3 \pm 5.8 \mathrm{~ms}$ (range, 3-121 ms). Subiculum pyramidal cells have been divided into two or more categories according to burst-firing characteristics; available evidence indicates that bursting cells are more common in subicular deep layers (for review, see Witter, 2006). We defined bursting cells as those with modal interspike intervals $\leq 8 \mathrm{~ms}$. This threshold resulted in a grouping of 11 bursting BVCs (modal ISI, $4.3 \pm 0.3 \mathrm{~ms}$ ) and 25 nonbursting BVCs (modal ISI, $46.0 \pm$ $\left.7.0 \mathrm{~ms} ; t_{(34)}=3.92 ; p=0.0004\right)$. Eight of the 11 bursting BVCs were located in the deeper half of the pyramidal cell layer, consistent with the idea that bursting cells are more common in deep subiculum. The bursting BVCs showed significantly higher global mean rates $(7.2 \pm 1.3 \mathrm{~Hz}$ ) than nonbursting BVCs (mean, $\left.4.1 \pm 0.6 \mathrm{~Hz} ; t_{(34)}=2.58 ; p=0.01\right)$ and significantly deeper theta modulation $(15.8 \pm 3.3)$ than nonbursting BVCs $\left(7.1 \pm 1.3 ; t_{(34)}=\right.$ 2.96; $p=0.006)$, which remained so after removing BVCs with global rates $\leq 2.0 \mathrm{~Hz}$ (bursters, $n=10$; theta modulation, $17.2 \pm$ 3.3 ; nonbursters, $n=18 ; 8.0 \pm 1.7 ; t_{(26)}=2.69 ; p=0.01$ ). (See supplemental material, available at www.jneurosci.org, for alternative analysis with similar results and representative examples of autocorrelograms and ISI histograms.)

\section{Discussion}

Thirty-six dorsal subicular pyramidal cells had spatial firing characteristics consistent with those predicted for BVCs (O'Keefe and Burgess, 1996; Burgess et al., 2000; Hartley et al., 2000). Twentyfour of these cells were recorded in several environments differing in boundary geometry and other nonspatial aspects of the recording context. Their firing fields reflect the distance and allocentric direction vector to the boundary and are relatively uninfluenced by the directional orientation of the animal, or the precise characteristics of the boundary and recording context. Neither the color, material, and nature (wall or drop) of the boundary, nor the surrounding distal cues (so long as the sense of direction is well anchored) have much influence on firing. Below, we discuss the properties of "boundaries" and compare the BVCs to other cell types.

\section{What constitutes a boundary?}

The main determinant of BVC firing is the vector from the rat to the boundary regardless of the color, material, or shape of the boundary. Most BVCs showed firing in the wall-less environments that was generally consistent with firing in the walled environments, suggesting that any given BVC responds to both extended vertical surfaces and to drops. Muller and Kubie (1987) first suggested that barriers controlled the firing of PCs, emphasizing the way in which impeding movement in a place field greatly decreased firing in that field. Our evidence suggests that boundaries may be defined by both sensory cues and limitations to movement. In wall-less environments (environments $\mathrm{c}$ and $\mathrm{d}$ ), there are two parallel sets of boundaries with rather different properties: the platform edge and $\sim 5-10 \mathrm{~cm}$ farther out, the limit of space reachable by leaning over the edge. Some cells appear to respond predominantly relative to the edge of the platform and others relative to the edge of reachable space. In addition, the drop between two raised platforms (Fig. $3 E$, environment $\mathrm{e}$ Apart) produces normal BVC firing although the rat can jump across it. Determining which sensory inputs drive BVC firing requires additional research; however, BVCs firing in complete darkness (Fig. 3C) indicate that they are not only visual, whereas BVCs firing offset from the boundary (Fig. $2 \mathrm{E}$ ) indicate that they are not only tactile.

Thus, a boundary is an abstract concept that may reflect sensory properties of environment features such as the sight or feel of a wall or an extended edge, as well as impediments to movement. Furthermore, the relative importance of these factors may vary across BVCs.

\section{Subicular BVCs are impervious to environmental changes which cause PC remapping}

Hippocampal PCs are well known to remap (Muller and Kubie, 1987) when the sensory qualities of two environments differ sufficiently. Generally, the greater the difference, the greater the remapping (Muller and Kubie, 1987; Shapiro et al., 1997; Lever et al., 2002a; Anderson and Jeffery, 2003; Wills et al., 2005). Sharp (1997, 1999) used walled enclosures and observed a relative absence of remapping in subiculum compared with CA1. In the present work, the difference between environment a vs environment $\mathrm{b}$ produced strong remapping in CA1 PCs without disrupting BVC firing. The more dramatic change to a wall-less environment (environment a vs environment c) produced strong remapping in CA1 $\mathrm{PCs}$ and also elicited remapping in many of the non-BVC subicular cells recorded simultaneously with BVCs (data not shown). However 14 of 17 BVCs were primarily insensitive even to this change. Together, the data strongly suggest that subicular BVCs remap less than PCs in response to the same environmental differences.

\section{Relationship of subicular BVCs to the wider hippocampal formation}

Environmental boundaries are also important determinants of firing in entorhinal cortex, and cells there are also less likely to remap than hippocampal PCs (Quirk et al., 1992; Savelli et al., 2008). Most relevant to the BVCs described here are recent reports of "border cells" (Solstad et al., 2008) or "putative boundary cells" (Savelli et al., 2008) in medial entorhinal cortex (mEC), cells that fire along one or more boundaries of the enclosure, regardless of the animal's directional orientation. These cells might simply be a subset of BVCs with short distance tuning. This would be consistent with the BVC model, according to which the subicular BVCs provide inputs to PCs in the hippocampus proper, the most obvious route being via entorhinal cortex. Solstad et al. (2008) made the alternative interpretation that the subicular BVCs reported by Barry et al. (2006) might be axons from entorhinal border cells. However, our results (Fig. 2) demonstrate that subicular BVCs have waveforms characteristic of principal cells. Our finding of many BVCs in proximal-to-CA1 argues against subicular BVCs simply reflecting input from entorhinal border cells. This is because border cells are found in $\mathrm{mEC}$ (Solstad et al., 2008) and the projection from the $\mathrm{mEC}$ to the subiculum is confined to the distal-to-CA1 part of the subiculum (Witter, 2006).

Our interpretation of subicular BVCs as an input to PC firing entails supplementing the classic view of subiculum as providing hippocampal output to extra-hippocampal regions by virtue of its massive input from CA1. Supporting this revision, we note (1) in some circumstances, subicular firing acts independently of changes to CA1 firing, most notably when CA1 strongly remaps and subicular cells do not (see Sharp, 1997, 1999; and our own data, above); (2) a physiologically active subiculum-entorhinalhippocampus circuit (Kloosterman et al., 2004); (3) strong sub- 
icular projections to parasubiculum and presubiculum, which both provide massive input to hippocampally projecting entorhinal cells (Witter and Amaral, 2004). Indeed, the parasubiculum's cortical input is mainly the subiculum, its output mainly the entorhinal cortex (Witter and Amaral, 2004). Thus, subicular output clearly (re)enters the hippocampal formation.

An important prediction of the BVC model is that short-range BVCs will be more numerous than long-range ones (Hartley et al., 2000) and this appears to be true of our subicular recordings (Fig 2). If medial entorhinal border cells turn out to be a subset of the more general class of BVCs, then longer-range BVCs should also be found in entorhinal cortex. A small number of cells consistent with longer-range BVCs were reported by Solstad et al. [(2008), their Fig. 1B, cell 677; their supplemental Fig. S4, cells 443 and 348], although we note their criteria for identifying border cells would exclude many longer-range BVCs. There are anecdotal observations of such cells in $\mathrm{mEC}$ from other laboratories [J. Krupic, unpublished (our group) and J. Csicsvari, personal communication]. It will be important to determine whether longer-range BVCs are sufficiently numerous in $\mathrm{mEC}$ to be compatible with the BVC model, or whether cells with responses strictly at the border predominate in $\mathrm{mEC}$.

Although some border cells may be short-range BVCs, Solstad et al. (2008) also report border cells that are not BVCs (e.g., cells that only fire along part of a wall, and cells that fire along all walls). Another potential difference concerns the response to wall removal. The fields of most subicular BVCs were not disrupted by removal of the boundary walls. In contrast, most of the entorhinal border cells tested without walls [ 8 of the 10 tested by Solstad et al. (2008), their supplemental Fig. S7] appear to remap when walls are removed (the firing field shifting from one wall to another or changing shape and size), although, like us, Solstad et al. (2008) took care to anchor the head direction system throughout (their supplemental Fig. S8).

In conclusion, we report subicular neurons whose spatial firing patterns correspond closely to the BVC predicted by models of the environmental inputs to hippocampal PCs. Several questions are raised by this finding: Where does the sensory information driving the firing of subicular BVCs come from? Does information from subicular BVCs reach hippocampal PCs, and if so, does it travel via the medial entorhinal border cells (Solstad et al., 2008)? Do the BVCs provide a complementary input to PCs to stabilize the path-integrative input from medial entorhinal grid cells (Hafting et al., 2005), as proposed by O'Keefe and Burgess (2005).

\section{References}

Anderson MI, Jeffery KJ (2003) Heterogeneous modulation of place cell firing by changes in context. J Neurosci 23:8827-8835.

Barry C, Burgess N (2007) Learning in a geometric model of place cell firing. Hippocampus 17:786-800.

Barry C, Lever C, Hayman R, Hartley T, Burton S, O’Keefe J, Jeffery K, Burgess N (2006) The boundary vector cell model of place cell firing and spatial memory. Rev Neurosci 17:71-97.
Burgess N, Jackson A, Hartley T, O'Keefe J (2000) Predictions derived from modelling the hippocampal role in navigation. Biol Cybern 83:301-312.

Cacucci F, Lever C, Wills TJ, Burgess N, O'Keefe J (2004) Theta-modulated place-by-direction cells in the hippocampal formation in the rat. J Neurosci 24:8265-8277.

Hafting T, Fyhn M, Molden S, Moser MB, Moser EI (2005) Microstructure of a spatial map in the entorhinal cortex. Nature 436:801-806.

Hartley T, Burgess N, Lever C, Cacucci F, O'Keefe J (2000) Modeling place fields in terms of the cortical inputs to the hippocampus. Hippocampus 10:369-379.

Karlsson MP, Frank LM (2008) Network dynamics underlying the formation of sparse, informative representations in the hippocampus. J Neurosci 28:14271-14281.

Kloosterman F, van Haeften T, Lopes da Silva FH (2004) Two reentrant pathways in the hippocampal-entorhinal system. Hippocampus 14:1026-1039.

Lever C, Wills T, Cacucci F, Burgess N, O'Keefe J (2002a) Long-term plasticity in hippocampal place-cell representation of environmental geometry. Nature 416:90-94.

Lever C, Burgess N, Cacucci F, Hartley T, O'Keefe J (2002b) What can the hippocampal representation of environmental geometry tell us about Hebbian learning? Biol Cybern 87:356-372.

Muller RU, Kubie JL (1987) The effects of changes in the environment on the spatial firing of hippocampal complex-spike cells. J Neurosci 7:1951-1968.

O'Keefe J, Burgess N (1996) Geometric determinants of the place fields of hippocampal neurons. Nature 381:425-428.

O'Keefe J, Burgess N (2005) Dual phase and rate coding in hippocampal place cells: theoretical significance and relationship to entorhinal grid cells. Hippocampus 15:853-866.

Quirk GJ, Muller RU, Kubie JL, Ranck JB Jr (1992) The positional firing properties of medial entorhinal neurons: description and comparison with hippocampal place cells. J Neurosci 12:1945-1963.

Rivard B, Li Y, Lenck-Santini PP, Poucet B, Muller RU (2004) Representation of objects in space by two classes of hippocampal pyramidal cells. J Gen Physiol 124:9-25.

Savelli F, Yoganarasimha D, Knierim JJ (2008) Influence of boundary removal on the spatial representations of the medial entorhinal cortex. Hippocampus 18:1270-1282.

Shapiro ML, Tanila H, Eichenbaum H (1997) Cues that hippocampal place cells encode: dynamic and hierarchical representation of local and distal stimuli. Hippocampus 7:624-642.

Sharp PE (1997) Subicular cells generate similar spatial firing patterns in two geometrically and visually distinctive environments: comparison with hippocampal place cells. Behav Brain Res 85:71-92.

Sharp PE (1999) Subicular place cells expand or contract their spatial firing pattern to fit the size of the environment in an open field but not in the presence of barriers: comparison with hippocampal place cells. Behav Neurosci 113:643-662.

Solstad T, Boccara CN, Kropff E, Moser MB, Moser EI (2008) Representation of geometric borders in the entorhinal cortex. Science 322:1865-1868.

Wills TJ, Lever C, Cacucci F, Burgess N, O'Keefe J (2005) Attractor dynamics in the hippocampal representation of the local environment. Science 308:873-876.

Witter MP (2006) Connections of the subiculum of the rat: topography in relation to columnar and laminar organization. Behav Brain Res 174:251-264.

Witter MP, Amaral DG (2004) Hippocampal formation. In: The rat nervous system (Paxinos G, ed), pp 635-704. London: Elsevier. 